\title{
Práticas realizadas pela equipe multidisciplinar em cuidados paliativos durante a pandemia COVID-19
}

\author{
Practices carried out by the multidisciplinary team in palliative care during the COVID-19 \\ pandemic
}

Prácticas realizadas por el equipo multidisciplinar en cuidados paliativos durante la pandemia COVID-19

\author{
Amanda Conrado Silva Barbosa \\ ORCID: https://orcid.org/0000-0003-2092-2099 \\ Universidade do Estado de Minas Gerais, Brasil \\ E-mail:amandaconradosb@hotmail.com \\ Caique Alves Rezende \\ ORCID: https://orcid.org/0000-0003-0956-7321 \\ Universidade do Estado de Minas Gerais, Brasil \\ E-mail: caiquerezende25@gmail.com \\ Amanda Cristina Teixeira do Prado \\ ORCID: https://orcid.org/0000-0003-3783-1695 \\ Universidade do Estado de Minas Gerais, Brasil \\ E-mail: amanda.teixeira.11@outlook.com \\ Yasmim Kelen Ferreira da Silva \\ ORCID: https://orcid.org/0000-0003-4779-4568 \\ Universidade do Estado de Minas Gerais, Brasil \\ E-mail: yferreira38@gmail.com \\ Débora Santos Ferreira \\ ORCID: https://orcid.org/0000-0001-7518-9950 \\ Universidade do Estado de Minas Gerais, Brasil \\ E-mail: psideborasantosferreira@gmail.com \\ Rangel Vinícius Xavier \\ ORCID: https://orcid.org/0000-0002-4385-6832 \\ Universidade do Estado de Minas Gerais, Brasil \\ E-mail: rangel.1693011@ discente.uemg.br \\ Bianca Camila Gonçalves Moreira \\ ORCID: https://orcid.org/0000-0002-3379-2513 \\ Universidade do Estado de Minas Gerais, Brasil \\ E-mail: bianca.1692238@discente.uemg.br \\ Leandro Victor Silva Ribeiro \\ ORCID: https://orcid.org/0000-0002-3502-6513 \\ Universidade do Estado de Minas Gerais, Brasil \\ E-mail: leandrovictorsr@gmail.com \\ Fernanda Oliveira de Assis \\ ORCID: https://orcid.org/0000-0002-4339-6274 \\ Universidade do Estado de Minas Gerais, Brasil \\ E-mail: fernanda.assis.d@gmail.com \\ Roseli Silva de Oliveira \\ ORCID: https://orcid.org/0000-0003-3006-493X \\ Universidade do Estado de Minas Gerais, Brasil \\ E-mail: roseli9957@gmail.com
}

\begin{abstract}
Resumo
O objetivo do presente estudo é identificar as evidências disponíveis na literatura sobre as práticas realizadas pela equipe multidisciplinar em cuidados paliativos durante a pandemia COVID-19. Refere-se a um estudo de revisão integrativa da literatura, realizada nas seguintes bases de dados: Base de dados de Enfermagem (BDENF), Medical Literature Analysis and Retrieval Sistem on-line (MEDLINE) e Literatura Latino-Americana e do Caribe em Ciências da Saúde (LILACS). Realizou-se busca temporal, de publicações entre os meses de dezembro de 2020 a janeiro de 2021. A amostra foi composta por nove artigos. As pesquisas selecionadas foram publicadas no ano de 2020, em idioma inglês, e a maior parte realizada nos Estado Unidos da América (77,8\%). Quanto ao delineamento dos estudos, nove (100\%) apresentavam abordagem quantitativa. Após a leitura e sumarização dos dados emergiram duas categorias, a saber: Práticas e cuidados dispensados aos pacientes e familiares durante a pandemia COVID-19 e Estratégias de enfrentamento pela equipe multidisciplinar de cuidados paliativos. Nesse sentido, o presente estudo
\end{abstract}


possibilitou a compreensão sobre a escassez de recursos físicos e humanos, observados durante o agravamento da pandemia causada pela COVID-19 e, consequentemente o impacto frente à continuidade das práticas realizadas pela equipe de cuidados paliativos.

Palavras-chave: Cuidados paliativos; Pessoal de saúde; Pandemias; Infecções por coronavírus.

\begin{abstract}
The aim of the present study is to identify the evidence available in the literature about the practices carried out by the multidisciplinary team in palliative care during the COVID-19 pandemic. It refers to an integrative literature review study carried out in the following databases: Nursing Database (BDENF), Medical Literature Analysis and Retrieval Online System (MEDLINE) and Latin American and Caribbean Literature in Science of Health (LILACS). A temporal search was carried out for publications between the months of December 2020 and January 2021. The sample consisted of nine articles. The selected surveys were published in 2020, in English, and most of them were carried out in the United States of America (77.8\%). As for the design of the studies, nine (100\%) had a quantitative approach. After reading and summarizing the data, two categories emerged, namely: Practices and care provided to patients and families during the COVID-19 pandemic and Coping strategies by the multidisciplinary palliative care team. In this sense, the present study made it possible to understand the scarcity of physical and human resources observed during the worsening of the pandemic caused by COVID-19 and, consequently, the impact on the continuity of practices carried out by the palliative care team.
\end{abstract}

Keywords: Palliative care; Health Personnel; Pandemics; Coronavirus infections.

\title{
Resumen
}

El objetivo del presente estudio es identificar la evidencia disponible en la literatura sobre las prácticas realizadas por el equipo multidisciplinario en cuidados paliativos durante la pandemia de COVID-19. Se refiere a un estudio de revisión integradora de la literatura realizado en las siguientes bases de datos: Base de datos de enfermería (BDENF), Sistema en línea de análisis y recuperación de literatura médica (MEDLINE) y Literatura latinoamericana y caribeña en ciencia de la salud (LILACS). Se realizó una búsqueda temporal de publicaciones entre los meses de diciembre de 2020 y enero de 2021. La muestra estuvo conformada por nueve artículos. Las investigaciones seleccionadas fueron publicadas en 2020, en inglés, y la mayoría de ellas se realizaron en los Estados Unidos de América (77,8\%). En cuanto al diseño de los estudios, nueve (100\%) tuvieron un enfoque cuantitativo. Después de leer y resumir los datos, surgieron dos categorías, a saber: Prácticas y cuidados brindados a pacientes y familiares durante la pandemia de COVID-19 y Estrategias de afrontamiento por parte del equipo multidisciplinario de cuidados paliativos. En este sentido, el presente estudio permitió comprender la escasez de recursos físicos y humanos observada durante el agravamiento de la pandemia por COVID-19 y, en consecuencia, el impacto en la continuidad de las prácticas realizadas por el equipo de cuidados paliativos.

Palabras clave: Cuidados paliativos; Personal sanitario; Pandemias; Infecciones por coronavirus.

\section{Introdução}

No mês de dezembro de 2019, houve na cidade de Wuhan, na China, um surto causado pelo novo coronavírus, SARSCOV-2. O vírus causa uma doença infecciosa que possui alta transmissibilidade, através da emissão de gotículas pela fala, tosse ou espirro. Sua apresentação clínica varia de sintomas leves gripais a casos com acometimento respiratório grave. Com o avanço da doença, ultrapassando barreiras geográficas, em 2020 foi considerada como pandemia (Heymann \& Shindo, 2020).

Houve, portanto, a necessidade de medidas restritivas para conter a propagação viral, através de fechamento de atividades consideradas não essenciais, isolamento social e uso contínuo de medidas de proteção individual como o uso de máscaras faciais, álcool em gel e lavagem das mãos (WHO, 2020). Contudo, após o afrouxamento dessas medidas e após um ano de pandemia, o Brasil se encontra entre os países com maiores índices de mortalidade pelo coronavírus (OPAS; OMS, 2021). Segundo o Ministério da Saúde (MS), até o mês de maio de 2021, o número total de casos confirmados somou 16.471.800 e de óbitos 461.057 .

A doença nomeada pela Organização Mundial da Saúde (OMS) como COVID-19 acomete principalmente grupos considerados mais vulneráveis como: idosos, portadores de comorbidades crônicas, obesos e imunossuprimidos pelo potencial aumentado de evolução para a síndrome respiratória aguda grave. Após a identificação do vírus foi possível apontar que sua virulência e patogenicidade estão diretamente ligadas à gama de proteínas virais (Ahn et al., 2020). O vírus possui um período de incubação, determinado pelo tempo de infecção e aparecimento de sintomas, entre cinco e oito dias (Zhang et al., 2020). 
Estudos apontam que devido à possibilidade da doença se apresentar de forma assintomática ou leve e da transmissão ocorrer antes do aparecimento de sintomas, há o aumento importante no potencial de contágio (Li et al., 2020; Zhang et al., 2020). Além disso, autores demonstram a efetividade do controle da infecção através do isolamento social, para conter a disseminação do vírus em uma determinada área, e consequentemente, diminuir o aparecimento de novos casos. Contudo, o controle da infecção viral somente é efetivo e duradouro através de programa amplo de vacinação que abarque diferentes faixas etárias, independentemente da presença de comorbidades (Zhang et al., 2020).

Devido ao aumento exponencial de atendimentos e internações de casos agravados pelo coronavírus em todo o mundo, houve grande impacto na prestação de serviços essenciais de saúde (WHO, 2021). Em estudo realizado em 105 países revelou que as interrupções dos serviços essenciais de saúde aconteceram em quase todos eles, sendo mais prevalentes em países subdesenvolvidos. Dentre os serviços de saúde afetados foram identificados, programas de atendimento as pessoas portadoras de doenças transmissíveis, doenças não transmissíveis, saúde mental, saúde reprodutiva, materna, neonatal, infantil e adolescente e serviços de nutrição (WHO, 2020).

No Brasil, observou-se o aumento progressivo de casos confirmados de COVID-19, altas taxas de infecção e de mortalidade, determinando a necessidade de abertura de novos leitos de terapia intensiva com maior demanda de insumos e contratação de mão de obra qualificada para atender um número cada vez maior de casos agravados pela infecção. Contudo, o sistema único de saúde se encontra em colapso, pela ineficiência e morosidade governamental, que por não conseguir atender a crescente demanda, acabou por provocar à sobrecarga das equipes, a presença de cargas horárias extenuantes, além de diversos profissionais infectados pelo coronavírus no ambiente laboral (Ribeiro et al., 2020).

Além do exposto, os profissionais de saúde deparam-se constantemente com difíceis decisões éticas sobre priorizar os cuidados para pacientes com maior chance de sobrevivência. A integração dos Cuidados Paliativos no planejamento da pandemia é essencial para assegurar cuidado de qualidade, enquanto se faz necessário decidir quem precisa ou não de cuidados intensivos para manutenção da vida (Fadul \& Bruera, 2021).

Para atender essa demanda e garantir a continuidade dos serviços preventivos e curativos principalmente para grupos mais vulneráveis, a OMS declarou a necessidade de ações direcionadas para reorganizar e manter o acesso a serviços de saúde essenciais, seguros e de alta qualidade (WHO, 2021). Neste sentido, a Academia Nacional de Cuidados Paliativos (ANCP) elaborou materiais para auxiliar profissionais e equipes de Cuidados Paliativos durante a pandemia. As ações devem ser ofertadas em conjunto com o tratamento padrão de qualquer doença que ameace a continuidade da vida, não devendo ser associado com a omissão ou exclusão, mesmo durante uma pandemia (ANCP, 2021).

Trata-se de abordagens voltadas para o controle de sintomas, conforto e qualidade de vida, executados por uma equipe multidisciplinar que garante o apoio e assistência para os pacientes e familiares, incluindo atendimento às necessidades básicas e apoio psicológico no momento do luto, reconhecidos no contexto dos direitos humanos (OMS, 2020).

Entretanto, estudos têm demonstrado que a pandemia pode afetar negativamente a continuidade dos Cuidados Paliativos, principalmente em relação aos pacientes portadores de morbidades crônicas. Dentre os fatores que dificultam a prestação da assistência, encontra-se a demora na busca de atendimento para emergências, como infarto cardíaco e derrame; aumento do risco de infecção em pessoas com insuficiência renal que precisam de tratamento em centros de diálise; crise diabética causada por interrupção no fornecimento de insulina; piora do prognóstico do câncer como resultado de um diagnóstico tardio e deterioração do quadro de pessoas com doenças respiratórias crônicas devido a interrupções na reabilitação pulmonar (OPAS, 2020).

Diante do exposto, verifica-se a necessidade de identificar as práticas e cuidados realizados pela equipe multidisciplinar em Cuidados Paliativos durante a pandemia COVID-19, a fim de sintetizar as pesquisas que versam sobre o conforto e cuidados dispensados ao paciente e sua família, bem como as práticas adotadas em casos confirmados e agravados 
pelo coronavírus. Espera-se que a equipe de Cuidados Paliativos promova práticas visando o alívio e conforto das dores dos pacientes e de seus familiares, de maneira humanizada, potencializando vínculos e garantindo a prestação do cuidado efetivo.

Portanto, o objetivo deste estudo é identificar estudos disponíveis na literatura sobre as práticas realizadas pela equipe multidisciplinar em Cuidados Paliativos durante a pandemia COVID-19.

\section{Metodologia}

Trata-se de uma revisão integrativa da literatura para a identificação de produções científicas sobre as práticas em Cuidados Paliativos realizadas em tempos de pandemia COVID-19. Esse estudo seguiu as seis etapas recomendadas para a elaboração de uma revisão integrativa de qualidade: 1) Identificação do tema e seleção da questão de pesquisa; 2) Estabelecimento dos critérios de inclusão e exclusão; 3) Identificação dos estudos pré-selecionados e selecionados; 4) Categorização dos estudos selecionados; 5) Análise e interpretação dos resultados; e 6) Apresentação da revisão/síntese do conhecimento (Gomes \& Caminha, 2014). Para concepção da pergunta norteadora, recorreu-se através da estratégia do acrômio PICO, em que (P) significa Paciente ou Problema, (I) Intervenção, (C) Comparação e (O) Desfecho (Santos et al., 2007). Portanto, para este estudo, definiu-se: (P) equipe multidisciplinar, (I) práticas em cuidados paliativos (O) durante a pandemia COVID-19. Dessa forma, a pergunta norteadora da pesquisa foi "quais as práticas em cuidados paliativos são realizadas pela equipe multidisciplinar em tempos de pandemia COVID-19?”.

O levantamento bibliográfico foi realizado entre os meses de dezembro de 2020 a janeiro de 2021, nas seguintes bases de dados: Base de dados de Enfermagem (BDENF), Medical Literature Analysis and Retrieval Sistem on-line (MEDLINE) e Literatura Latino-Americana e do Caribe em Ciências da Saúde (LILACS). Foram utilizados, para realizar a pesquisa, os utilizando-se Descritores em Ciências da Saúde (DeCs) "Cuidados paliativos"; "Pessoal de Saúde"; "Pandemias"; "Infecções por Coronavírus" intermediados pelos operadores booleanos "AND", e "OR", a fim de aumentar o escopo da busca.

Definiu-se como critérios de inclusão: artigos disponíveis na integra, com acesso livre e publicados nos idiomas português, inglês ou espanhol e que abordassem a temática pesquisada. Foram excluídos os artigos que não atenderam os propósitos da pesquisa, artigos incompletos, teses, dissertações, publicações duplicadas e artigos anteriores ao ano de 2020. Justifica-se o critério de busca temporal, pela descoberta recente da infecção causada pelo coronavírus e a definição da pandemia ocorrer no ano de 2020.

Para a coleta de dados e, posteriormente, caracterização das produções selecionadas, utilizou-se um instrumento elaborado pelos autores, contemplando os seguintes itens: título em inglês e português, autores, ano da publicação, país de origem do estudo, idioma da publicação, objetivo, delineamento do estudo, nível de evidência, qualis do periódico e principais resultados.

Procedeu-se a análise de forma crítica, buscando a identificação dos achados nos diferentes estudos. Assim, através de revisores elencados entre os autores deste estudo, foi realizada à organização e tabulação dos dados, utilizando-se estatística descritiva. Posteriormente, ocorreu a discussão com interpretação e eleição de duas categorias de análise, identificando-se as implicações para a prática em cuidados paliativos em tempos de pandemia COVID-19 e as estratégias de enfrentamento pela equipe multidisciplinar.

\section{Resultados}

Tendo por referência a questão norteadora deste estudo e a busca nas bases de dados realizada para coleta de material, foram encontrados 38 artigos com a temática proposta. Em seguida, fez-se a leitura dos títulos e resumos, considerando-se os critérios de seleção descritos no fluxograma abaixo. Essa etapa resultou na designação de nove artigos que foram lidos na íntegra. 
Figura 1 - Fluxograma do processo de seleção dos artigos nas bases de dados.

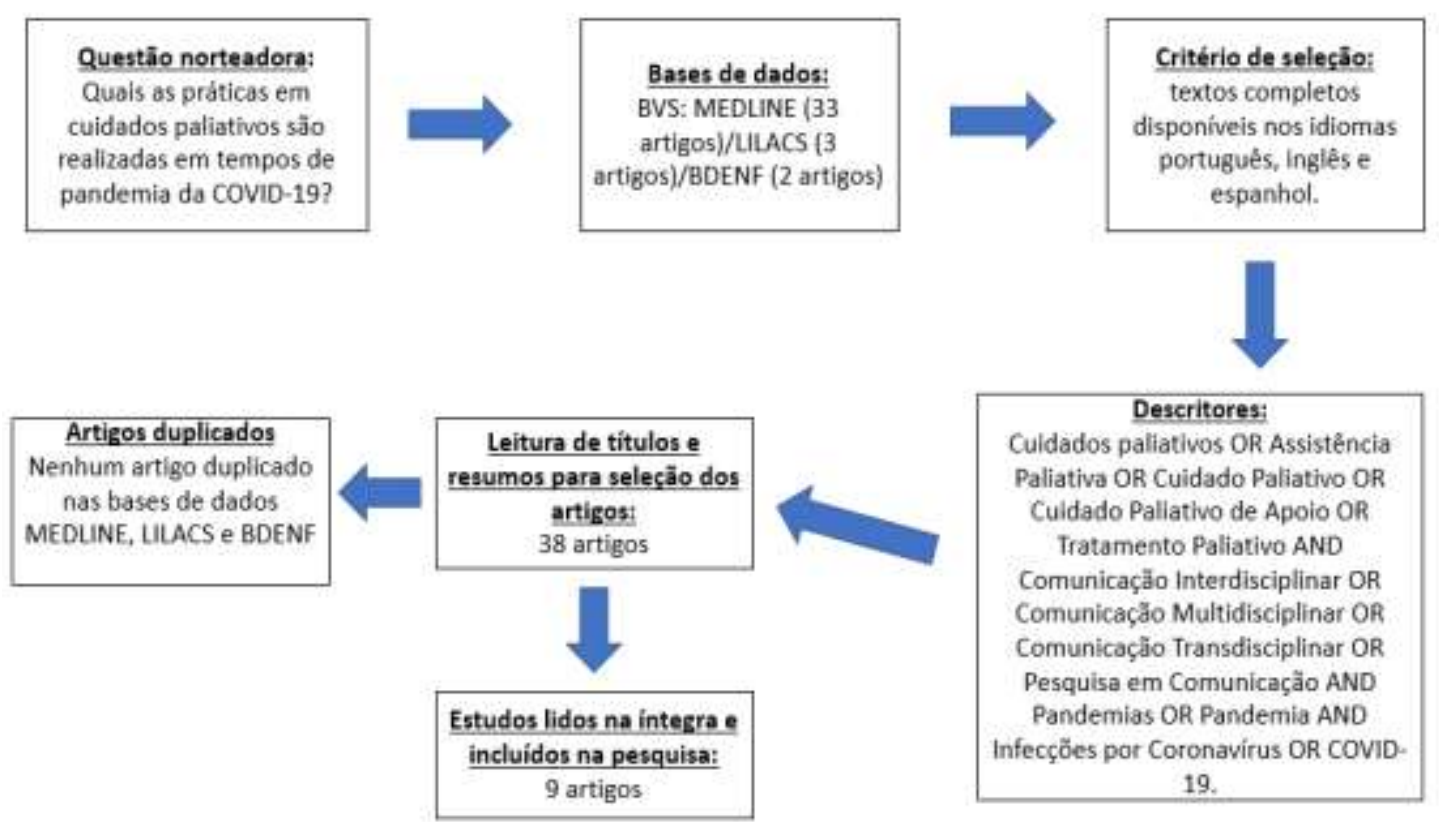

Fonte: Autores (2021).

A amostra desta revisão constitui-se de nove artigos em seu total, sendo oito publicados na base de dados Medical Literature Analysis and Retrieval System Online (MEDLINE) e um artigo publicado na base de dados Literatura LatinoAmericana e do Caribe em Ciências da Saúde (LILACS).

Os artigos selecionados foram publicados no ano de 2020, em idioma inglês, sendo que um $(11,1 \%)$ dos estudos foi realizado no Brasil, um $(11,1 \%)$ no Reino Unido e os sete $(77,8 \%)$ restantes nos Estado Unidos da América.

Quanto ao delineamento dos estudos, nove (100\%) apresentavam abordagem quantitativa; com quatro $(44,4 \%)$ quase experimentais, três $(33,3 \%)$ não experimentais correlacionais, um $(11,1 \%)$ experimental e somente um $(11,1 \%)$ apresentava-se enquanto estudo observacional descritivo.

Os artigos selecionados foram descritos no quadro abaixo quanto à identificação (título no idioma inglês e português, autores, ano de publicação, país de origem do estudo e idioma), abordagem metodológica (compreendendo informações sobre o objetivo, base de dados, delineamento do estudo, nível de evidência e qualis do periódico) e síntese dos resultados encontrados (Quadro 1): 
Quadro 1 - Identificação, abordagem metodológica e síntese dos resultados dos artigos selecionados:

\begin{tabular}{|c|c|c|c|c|}
\hline $\begin{array}{c}\text { Título } \\
\text { (Inglês e Português) }\end{array}$ & $\begin{array}{c}\text { Autores } \\
\text { (ano)/país/idioma }\end{array}$ & Objetivo & $\begin{array}{c}\text { Delineamento/ní } \\
\text { vel de } \\
\text { evidência/qualis } \\
\text { do periódico }\end{array}$ & Resultados \\
\hline $\begin{array}{l}\text { COVID-19 and Hospital Palliative } \\
\text { Care - A service evaluation } \\
\text { exploring the symptoms and } \\
\text { outcomes of } 186 \text { patients and the } \\
\text { impact of the pandemic on } \\
\text { specialist Hospital Palliative Care } \\
\text { COVID-19 e Cuidados Paliativos } \\
\text { Hospitalares - Uma avaliação de } \\
\text { serviço que explora os sintomas e } \\
\text { resultados de } 186 \text { pacientes e o } \\
\text { impacto da pandemia em cuidados } \\
\text { paliativos de hospitais } \\
\text { especializados. }\end{array}$ & $\begin{array}{l}\text { Hetherington, L, et } \\
\text { al., } \\
(2020) \\
\text { EUA } \\
\text { Inglês }\end{array}$ & $\begin{array}{l}\text { Realizar o levantamento de } \\
\text { dados acerca dos sintomas de } \\
186 \text { pacientes que testaram } \\
\text { positivo para a COVID-19, a } \\
\text { fim caracterizar a forma de } \\
\text { tratamento e o manejo } \\
\text { sintomáticos realizados pela } \\
\text { equipe de Cuidados } \\
\text { Paliativos em hospitais } \\
\text { especializados. }\end{array}$ & $\begin{array}{l}\text { Estudo quase } \\
\text { experimental } \\
\text { Nível III } \\
\text { Qualis A1 }\end{array}$ & $\begin{array}{l}\text { Concluiu-se que os pacientes } \\
\text { testados positivos para a COVID- } \\
19 \text { apresentaram alto grau de } \\
\text { comorbidade e baixa expectativa } \\
\text { de vida. Também houve a } \\
\text { evidenciação de maneira frequente } \\
\text { dos sintomas de dispneia e } \\
\text { agitação, tratadas pela dosagem de } \\
\text { opióides e benzodiazepínicos. }\end{array}$ \\
\hline $\begin{array}{l}\text { Pediatric Palliative Care When } \\
\text { COVID-19 Positive Adults Are } \\
\text { Dying in a Children's Hospital } \\
\text { Cuidados paliativos pediátricos } \\
\text { quando adultos positivos para } \\
\text { COVID-19 estão morrendo em um } \\
\text { hospital infantil. }\end{array}$ & $\begin{array}{l}\text { Norris, S E; Strumph, } \\
\text { K; Rahmani N E, } \\
(2020) \\
\text { EUA } \\
\text { Inglês }\end{array}$ & $\begin{array}{l}\text { Desenvolver e potencializar a } \\
\text { atuação da equipe de } \\
\text { Cuidados Paliativos durante a } \\
\text { pandemia do COVID-19, } \\
\text { para que de forma } \\
\text { humanizada os pacientes e os } \\
\text { familiares, isentos de contato } \\
\text { social, possuíssem apoio na } \\
\text { transmissão de feedbacks } \\
\text { individuais, atualizações de } \\
\text { prontuários, além de } \\
\text { desenvolver uma escuta de } \\
\text { apoio nos momentos de } \\
\text { incertezas e enlutamento. }\end{array}$ & $\begin{array}{l}\text { Estudo } \\
\text { experimental } \\
\text { Nível II } \\
\text { Qualis A1 }\end{array}$ & $\begin{array}{l}\text { Evidenciou-se que o modelo de } \\
\text { ampliação e qualificação da equipe } \\
\text { de Cuidados Paliativos foi efetiva } \\
\text { para o enfrentamento e alívio das } \\
\text { dores dos pacientes e familiares } \\
\text { durante esse período pandêmico. } \\
\text { Além disso, auxiliou no combate } \\
\text { ao isolamento e a busca da } \\
\text { amenização dos impactos gerados } \\
\text { pela COVID-19. }\end{array}$ \\
\hline $\begin{array}{l}\text { Palliative care for patients with } \\
\text { severe covid-19 } \\
\text { Cuidados paliativos para pacientes } \\
\text { com covid-19 grave. }\end{array}$ & $\begin{array}{l}\text { Ting, R, et al., } \\
(2020) \\
\text { Reino Unido } \\
\text { Inglês }\end{array}$ & $\begin{array}{l}\text { Apresentar descritivamente a } \\
\text { atuação dos Cuidados } \\
\text { Paliativos em pacientes com } \\
\text { COVID-19, auxiliando no } \\
\text { manejo dos sintomas como a } \\
\text { agitação e ansiedade, além de } \\
\text { buscar amenizar as angústias } \\
\text { do distanciamento social por } \\
\text { meio da comunicação direta e } \\
\text { clara, sobretudo no processo } \\
\text { de luto. }\end{array}$ & $\begin{array}{l}\text { Estudo quase } \\
\text { experimental } \\
\text { Nível III } \\
\text { Qualis B5 }\end{array}$ & $\begin{array}{l}\text { Foi demonstrado que as formas de } \\
\text { atuação da equipe de Cuidados } \\
\text { Paliativos apresentam, de maneira } \\
\text { humanizada, estratégias que visem } \\
\text { tranquilizar pacientes com } \\
\text { COVID-19 e seus familiares. Para } \\
\text { isso, foi reconhecido as } \\
\text { especificidades de cada caso, o } \\
\text { cuidado no manejo dos sintomas, } \\
\text { das angústias e na transmissão de } \\
\text { informações de forma sensível. }\end{array}$ \\
\hline $\begin{array}{l}\text { The Role of Palliative Care in } \\
\text { Caring for the Families of Patients } \\
\text { With COVID- } 19 \text {. } \\
\text { O papel dos cuidados paliativos no } \\
\text { cuidado de famílias de pacientes } \\
\text { com COVID-19. }\end{array}$ & $\begin{array}{l}\text { Bakar, M, et al., } \\
(2020) \\
\text { EUA } \\
\text { Inglês }\end{array}$ & $\begin{array}{l}\text { Demonstrar a importância do } \\
\text { trabalho da equipe de } \\
\text { Cuidados Paliativos com os } \\
\text { familiares de pacientes } \\
\text { acometidos pela COVID-19, } \\
\text { auxiliando na transmissão de } \\
\text { informações e buscando } \\
\text { amenizar a angústia e o } \\
\text { sofrimento dos mesmos. }\end{array}$ & $\begin{array}{l}\text { Não } \\
\text { experimental } \\
\text { correlacional } \\
\text { Nível IV } \\
\text { Qualis B1 }\end{array}$ & $\begin{array}{l}\text { Concluiu-se que a elaboração do } \\
\text { plano multiprofissional para o } \\
\text { acolhimento das famílias de } \\
\text { pacientes com COVID-19, foi } \\
\text { crucial para potencializar os } \\
\text { vínculos entre a equipe de } \\
\text { Cuidados Paliativos e os familiares } \\
\text { dos infectados, além de } \\
\text { proporcionar uma relação de } \\
\text { confiança, cuidado e aproximação, } \\
\text { mesmo que de forma virtual. }\end{array}$ \\
\hline $\begin{array}{l}\text { What Should Palliative Care's } \\
\text { Response Be to the COVID-19 } \\
\text { Pandemic? } \\
\text { Qual deve ser a resposta dos } \\
\text { cuidados paliativos à pandemia do } \\
\text { COVID-19? }\end{array}$ & $\begin{array}{l}\text { Powell, V D; } \\
\text { Silveira, M J, } \\
(2020) \\
\text { EUA } \\
\text { Inglês }\end{array}$ & $\begin{array}{l}\text { Apontar as novas estratégias } \\
\text { das equipes de Cuidados } \\
\text { Paliativos para conseguir } \\
\text { responder ao aumento } \\
\text { progressivo de pacientes com } \\
\text { a COVID-19, objetiva-se } \\
\text { identificar as modificações } \\
\text { dentro da equipe e na forma } \\
\text { de atendimento dessas }\end{array}$ & $\begin{array}{l}\text { Não } \\
\text { experimental } \\
\text { correlacional } \\
\text { Nível IV } \\
\text { Qualis B1 }\end{array}$ & $\begin{array}{l}\text { Demonstrou-se que a mobilização } \\
\text { da equipe de Cuidados Paliativos } \\
\text { durante a pandemia do COVID-19 } \\
\text { foi importante para a manutenção } \\
\text { de comunicação com as famílias a } \\
\text { fim de mantê-las informadas, além } \\
\text { de oferecer equipes de suporte de } \\
\text { vida e do painel de morte, que se } \\
\text { apresentaram como essenciais no } \\
\text { apoio durante o tratamento dos }\end{array}$ \\
\hline
\end{tabular}




\begin{tabular}{|c|c|c|c|c|}
\hline & & pessoas. & & pacientes e no período de luto. \\
\hline 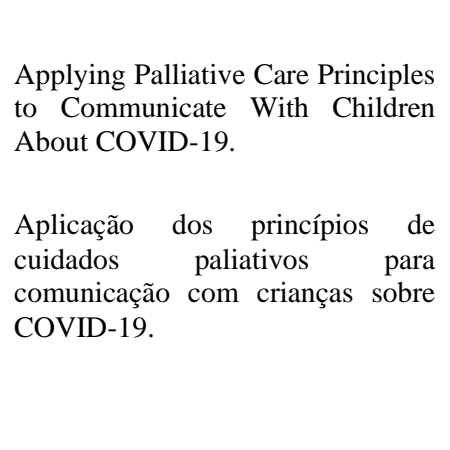 & $\begin{array}{l}\text { Weaver, M S; } \\
\text { Wiener L, } \\
(2020) \\
\text { EUA } \\
\text { Inglês }\end{array}$ & $\begin{array}{l}\text { Apontar os impactos que a } \\
\text { pandemia do COVID-19 } \\
\text { pode causar nas crianças e a } \\
\text { maneira que as equipes de } \\
\text { cuidados paliativos podem } \\
\text { gerir essas consequências } \\
\text { com os pacientes na faixa } \\
\text { etária infantil e seus } \\
\text { familiares. }\end{array}$ & $\begin{array}{l}\text { Estudo quase } \\
\text { experimental } \\
\text { Nível III } \\
\text { Qualis B1 }\end{array}$ & $\begin{array}{l}\text { Foi demonstrado que os cuidados e } \\
\text { ações em Cuidados Paliativos } \\
\text { possuem a capacidade de } \\
\text { proporcionar conforto para as } \\
\text { crianças e seus familiares, durante } \\
\text { a pandemia do coronavírus, o que } \\
\text { foi evidenciado pela necessidade } \\
\text { de resgate dos vínculos afetivos, a } \\
\text { sensibilidade sobre o momento de } \\
\text { incertezas e dores, além do papel } \\
\text { crucial de manter as medidas de } \\
\text { segurança para evitar a } \\
\text { contaminação. }\end{array}$ \\
\hline $\begin{array}{l}\text { Creating a Palliative Care Inpatient } \\
\text { Response Plan for COVID-19 - } \\
\text { The UW Medicine Experience } \\
\text { Criação de um plano de resposta a } \\
\text { pacientes internados em cuidados } \\
\text { paliativos para COVID-19 - A } \\
\text { experiência da medicina UW. }\end{array}$ & $\begin{array}{l}\text { Fausto, J, et al., } \\
(2020) \\
\text { EUA } \\
\text { Inglês }\end{array}$ & 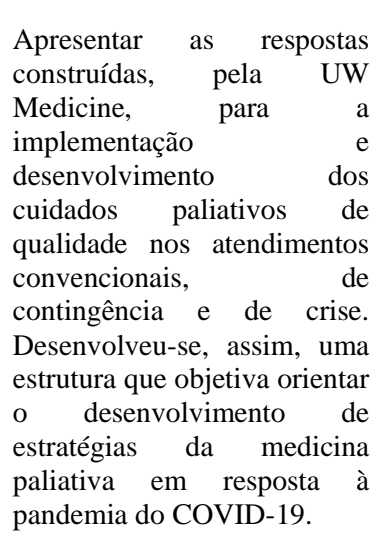 & $\begin{array}{l}\text { Estudo não } \\
\text { experimental } \\
\text { correlacional } \\
\text { Nível IV } \\
\text { Qualis B1 }\end{array}$ & $\begin{array}{l}\text { Conclui-se que os cuidados } \\
\text { paliativos podem se apresentar } \\
\text { como uma importante resposta de } \\
\text { auxílio e tratamento frente ao } \\
\text { coronavírus. A estratégia ainda se } \\
\text { encontra em desenvolvimento e } \\
\text { atualiza-se conforme as mudanças } \\
\text { e expansão do vírus pelo mundo. } \\
\text { Contudo, os cuidados paliativos de } \\
\text { alto nível, em situações de } \\
\text { contingência e crise, contribuem } \\
\text { para minimizar a quantidade de } \\
\text { procedimentos impróprios que se } \\
\text { relacionam com } \\
\text { desenvolvimento, adaptação e } \\
\text { implementação de estratégias. }\end{array}$ \\
\hline $\begin{array}{l}\text { Characteristics, } \\
\text { Management, and Outcomes of } \\
101 \text { Patients With COVID-19 } \\
\text { Referred for Hospital Palliative } \\
\text { Care. } \\
\text { Características, gerenciamento de } \\
\text { sintomas e resultados de } 101 \\
\text { pacientes com COVID-19 } \\
\text { encaminhados para cuidados } \\
\text { paliativos hospitalares. }\end{array}$ & $\begin{array}{l}\text { Lovell, N, et al., } \\
(2020) \\
\text { EUA } \\
\text { Inglês }\end{array}$ & $\begin{array}{l}\text { Apresentar o mapeamento de } \\
\text { sintomas de } 101 \text { pacientes } \\
\text { com COVID-19 que foram } \\
\text { encaminhados para hospitais } \\
\text { de cuidados paliativos. Por } \\
\text { meio da apresentação dos } \\
\text { quadros analisados objetiva- } \\
\text { se identificar a carga dos } \\
\text { sintomas e o seu manejo, as } \\
\text { respostas ao tratamento e os } \\
\text { resultados dos casos que } \\
\text { foram tratados à luz dos } \\
\text { cuidados paliativos. }\end{array}$ & $\begin{array}{l}\text { Estudo quase } \\
\text { experimental } \\
\text { Nível III } \\
\text { Qualis B1 }\end{array}$ & $\begin{array}{l}\text { Evidenciou-se forte relação dos } \\
\text { cuidados paliativos para o } \\
\text { enfrentamento da pandemia do } \\
\text { COVID-19. Identificou-se a } \\
\text { preponderância da falta de ar e } \\
\text { agitação nos quadros sintomáticos } \\
\text { dos pacientes, controlados com } \\
\text { utilização de pequenas doses de } \\
\text { opióides e benzodiazepínicos, por } \\
\text { infusão subcutânea. Contudo, } \\
\text { ainda é necessária uma maior } \\
\text { produção de pesquisas para } \\
\text { auxiliar no manejo dos sintomas e } \\
\text { nas formas de tratamento dos } \\
\text { infectados. }\end{array}$ \\
\hline $\begin{array}{l}\text { Palliative Renal Care and the } \\
\text { Covid- } 19 \text { Pandemic. } \\
\text { Cuidados renais paliativos e a } \\
\text { pandemia de Covid- } 19 \text {. }\end{array}$ & $\begin{array}{l}\text { Santos, C G S da, } \\
\text { et al., } \\
(2020) \\
\text { Brasil } \\
\text { Inglês }\end{array}$ & $\begin{array}{l}\text { Objetiva-se evidenciar as } \\
\text { estratégias da medicina } \\
\text { paliativa frente aos pacientes } \\
\text { nefropatas durante a } \\
\text { pandemia do coronavírus. } \\
\text { Expõem-se a importância da } \\
\text { tomada de decisão } \\
\text { compartilhada, do manejo de } \\
\text { sintomas, das habilidades em } \\
\text { comunicação e assistência ao } \\
\text { luto, para proporcionar o } \\
\text { melhor cuidado durante o } \\
\text { isolamento social. }\end{array}$ & $\begin{array}{l}\text { Estudo } \\
\text { observacional } \\
\text { descritivo } \\
\text { Nível IV } \\
\text { Qualis B2 }\end{array}$ & $\begin{array}{l}\text { Conclui-se que os Cuidados } \\
\text { Paliativos apresentam-se como } \\
\text { importante possibilidade de } \\
\text { cuidado e acolhimento dos } \\
\text { pacientes com disfunção renal, } \\
\text { visto que esses são considerados } \\
\text { parte do grupo de risco. Torna-se } \\
\text { importante colocar em pauta o } \\
\text { gerenciamento do seu tratamento, } \\
\text { seja no manejo dos sintomas } \\
\text { quanto na amenização das } \\
\text { angústias e dores presentes em } \\
\text { todas as dimensões do ser humano. }\end{array}$ \\
\hline
\end{tabular}

Fonte: Autores (2021). 


\section{Discussão}

Após a leitura e sumarização dos dados emergiram duas categorias, a saber: Práticas e cuidados dispensados aos pacientes e familiares durante a pandemia COVID-19; e Estratégias de enfrentamento pela equipe multidisciplinar de cuidados paliativos.

\section{Práticas e cuidados dispensados aos pacientes e familiares durante a pandemia COVID-19}

De acordo com estudo realizado por Ting e colaboradores (2020), os Cuidados Paliativos são comumente mal interpretados, por serem erroneamente compreendidos como assistência relevante apenas para indivíduos que estão em processo de terminalidade. Dessa forma, torna-se necessário a reafirmação dos cuidados paliativos como ações que permitem o alívio do sofrimento, por meio da prestação de cuidados holísticos e compassivos, que garanta o cuidado mais humanizado para pacientes que possuem doenças que podem ser fatais. Dessa forma, há estudos que destacam sobre a convergência em relação à implementação de protocolos medicamentosos para manejos de sintomas comumente observados como a angústia respiratória, caracterizada por falta de ar e agitação (Lovell et al., 2020; Ting et al., 2020; Hetherington et al., 2020).

Para a qualidade da assistência prestada aos pacientes, torna-se necessário o correto manejo dos sintomas apresentados pelos pacientes. Com isso, destaca-se o estudo realizado por Lovell et al (2020) que em seus achados, relatou que grande parte dos pacientes em Cuidados Paliativos hospitalares com COVID-19 apresentavam falta de ar e agitação, sendo nesses casos considerada eficaz a utilização de substâncias opioides e benzodiazepínicos.

Estudo concomitante de Ting et al (2020) e Hetherington et al (2020) também corroboram com esses achados, ao abordarem sobre a utilização de medicações na redução da ansiedade, agitação e angústia provocadas pelo isolamento familiar e sintomas respiratórios. Além desses sintomas, alguns pacientes apresentaram delirium, devido a situações de estresse. Dessa forma, estudos ressaltam sobre a utilização de antipsicóticos como o haloperidol, associado ou não aos benzodiazepínicos (Ting et al., 2020).

Não obstante a incerteza, insegurança e medo que o novo cenário implica, questões técnicas e instrumentais também surgiram nesse recorte. Dois pontos foram levantados pelos estudos utilizados como referência: a escassez de equipamentos de proteção individual (EPI) e o número reduzido de profissionais de Cuidados Paliativos. Observa-se, portanto, o desafio em garantir a segurança de todos os profissionais, principalmente daqueles que prestam técnicas em Cuidados Paliativos. Além da garantia institucional e trabalhista em proporcionar quantitativo suficiente de EPI's, há também à necessidade de expansão e disseminação dos conhecimentos e técnicas em Cuidados Paliativos para o maior número de funcionários envolvidos no cuidados, buscando atender e disseminar a filosofia dos Cuidados Paliativos para os pacientes e familiares, de maneira ética e responsável (Powell \& Silveira, 2020).

Em alguns momentos, os profissionais de saúde sentiram-se impotentes diante da situação irremediável de alguns pacientes. A sensação de despreparo e insegurança ao se depararem com demandas psicológicas, também foi relatada por alguns profissionais, visto que muitos tiveram uma formação tecnológica e curativista (Silva et al., 2021). Os Cuidados Paliativos ganham destaque nesse cenário, colocando em evidência a importância do cuidado multidisciplinar e o valor que as trocas de conhecimentos entre os profissionais podem contribuir para o cuidado prestado. Destaca-se também, a importância do cuidado integral ao paciente, colocando suas vontades e escolhas em questão, atuando não somente para este, mas também com seus familiares e a própria equipe envolvida na assistência (Norris \& Rahmani, 2020).

Nesta perspectiva, destaca-se a implementação de novos meios de comunicação utilizados durante o processo de internação de pacientes graves, visando â comunicação entre pacientes e familiares. Recursos áudio visuais como chamadas de vídeo, permitem a diminuição de distâncias físicas impostas durante o período pandêmico. Com o intuito de prestar assistência a esses familiares em momentos de luto, destaca-se a importância da equipe de Cuidados Paliativos em manter a família 
informada sobre o quadro do paciente, fornecendo mais conforto, por meio do apoio emocional e espiritual, respeitando sempre os desejos e vontades dos pacientes e seus familiares. Dessa forma, é inerente o uso da comunicação para tomada de decisões, disponibilização de informações claras por parte dos profissionais e comunicação terapêutica em momentos de terminalidade (Florêncio et al., 2020; Tritany et al., 2020).

\section{Estratégias de enfrentamento pela equipe multidisciplinar de Cuidados Paliativos}

Os artigos estudados também levantam a questão sobre a rápida progressão da doença, levando à incapacidade de decisões do paciente e à possível morte, o que demanda um curto tempo de prestação dos Cuidados Paliativos. Ressalta-se que a doença provocada pelo novo coronavírus possui alta capacidade de progressão, fazendo com que os pacientes gravemente enferos se tornem incapazes de tomar decisões. Portanto, torna-se fundamental a escuta ativa dos pacientes e seus familiares, buscando sempre compreender os desejos e vontades, minimizando as angústias provocadas por momentos de crise, além de respeitar as escolhas individuais, evitando o uso desnecessário e não desejado de medidas extremas para a manutenção da vida (Ting et al., 2020).

Como segundo ponto convergente existe a dificuldade de comunicação presencial, provocada pela alta capacidade de propagação do vírus causador da COVID-19, que fez com que medidas preventivas não farmacológicas fossem adotadas com o intuito de diminuir a disseminação da doença, como o distanciamento social, que acabou por impossibilitar o contato entre familiares, profissionais e amigos mais próximos. Com isso, viu-se a necessidade da implementação de estratégias que possibilitassem a comunicação de forma virtual, para o acesso rápido e eficaz a informação, além de proporcionar conexão dos pacientes com os seus familiares e auxílio na tomada de decisões (Ting et al., 2020; Powell \& Silveira, 2020; Bakar et al., 2020; Santos et al., 2020).

Assim, é importante destacar sobre a utilização do Teleatendimento por profissionais de saúde aos pacientes menos graves, com o intuito de priorizar atendimentos presenciais para pacientes mais críticos. Além disso, vale ressaltar sobre a relevância do estudo realizado por Wong et al (2020), que abordou sobre a importância da utilização de Teleconsultas com idosos, com o objetivo de amenizar sentimentos como a solidão provocados pelo isolamento social. As Teleconsultas são estratégias viáveis por serem intervenções de fácil acesso e responsáveis por promover satisfação para aqueles que residem em Instituições de Longa Permanência e que estão em cuidados em longo prazo (Yeung et al., 2020; Palliative \& Only, 2020). Estratégias como essa, são utilizadas também em ambientes intra-hospitalares para evitar o contato direto dos profissionais com pacientes contaminados, através da prestação da assistência por meio de telefonemas e videoconferências (Powell \& Silveira, 2020).

A tomada de decisões por familiares também requer uma atenção especial pela equipe de Cuidados Paliativos nesse momento pandêmico. A necessidade de se tomar a decisão por si só já é geradora de angústias por colocar o familiar em posição de detentor de decisões para um ente querido, ao saber da iminência da morte. A pandemia causada pela COVID 19 acabou por tornar o processo de tomada de decisões, um momento extremamente conturbado para os familiares, pois as famílias, na maior parte do tempo, não estão dentro do ambiente hospitalar junto com o enfermo devido às restrições que os protocolos aconselham. Com isso, desafios referentes à tomada de decisões são esperados, pois o afastamento provocado pelo isolamento social fez com que os familiares desconhecessem a gravidade da doença vivenciada pelos seus entes queridos, o que evidenciou a importância de medidas antecipatórias e o intenso apoio aos familiares durante esse momento de crise (Bakar et al., 2020).

Nessa perspectiva, evidencia-se a necessidade da criação de estratégias que possibilitem a prestação de cuidados de qualidade para aqueles pacientes que estão em Cuidados Paliativos, compreendendo que é um momento que gera dúvidas, angústias e sofrimento por se tratar de situações nunca antes vivenciados. Portanto, para a eficácia das estratégias 
implementadas é necessário que haja a disseminação e circulação das mesmas no meio científico e hospitalar, a fim de contribuir para a organização e prestação do serviço em larga escala, bem como proporcionar trocas de experiências e saberes entre a equipe prestadora do cuidado (Powell \& Silveira, 2020; Fausto et al., 2020).

Além disso, destaca-se também a realização de treinamentos considerados eficazes para profissionais que atuam em Cuidados Paliativos, a divisão de trabalhos e setores, a realização de grupos de ajuda e acolhimento, seja para familiares ou para os profissionais, além da importância da escuta ativa, comunicação e acolhimento adequado para esse momento (Araújo \& Silva, 2011).

\section{Conclusão}

As evidências encontradas nos estudos apontam que os Cuidados Paliativos são abordagens voltadas para o controle de sintomas, conforto e qualidade de vida de indivíduos que se encontram em situação ameaçadora de vida. Os cuidados prestados devem ser oferecidos em conjunto com o tratamento padrão de qualquer doença que ameace a continuidade da vida e, portanto, não devem ser associadas com a omissão ou abandono terapêutico, mesmo em tempos de pandemia. Além do mais, é importante destacar que as práticas dos Cuidados Paliativos são constituídas por pilares importantes para a eficácia do atendimento prestado, como a comunicação, controle dos sintomas, trabalho em equipe e apoio à família. No contexto da COVID-19, esses elementos ganharam mais ênfase, uma vez que os princípios dos Cuidados Paliativos visam uma assistência humana, integral e digna, não somente aos pacientes, mas aos seus familiares que se encontram afastados devido as restrições e controle da infecção.

Nesse sentido, o presente estudo possibilitou a compreensão sobre a escassez de recursos físicos e humanos, observados durante o agravamento da pandemia causada pela COVID-19 e, consequentemente o impacto frente à continuidade das práticas realizadas pela equipe de Cuidados Paliativos. Práticas encontradas na literatura consultada, como a referente ao acesso e prioridade ao tratamento para alguns pacientes em detrimento de outros, mostrou-se infelizmente, necessária devido ao colapso vivenciado pelos serviços de saúde. Vale ressaltar que os Cuidados Paliativos devem fornecer qualidade de vida aos pacientes, por meio do cuidado integral e humanizado proporcionando sensações de bem-estar, satisfação e autonomia. Ressalta-se que as limitações para a realização do estudo referem-se à escassez de pesquisas que abordassem as práticas e estratégias realizadas pela equipe multidisciplinar em Cuidados Paliativos durante a pandemia COVID-19. Ademais, visando elucidar as indagações, torna-se necessária a produção de estudos amplos que abordem as especificidades e limitações da prestação de cuidados e assistência aos pacientes em Cuidados Paliativos durante a pandemia causada pelo coronavírus humano.

\section{Referências}

Ahn, D. G., Shin, H. J., Kim, M. H., Lee, S., Kim, H. S., Myoung, J., Kim, B. T., \& Kim, S. J. (2020). Current status of epidemiology, diagnosis, therapeutics, and vaccines for novel coronavirus disease 2019 (COVID-19). Journal of Microbiology and Biotechnology, 30(3), 313-324. https://doi.org/10.4014/jmb.2003.03011

ANCP (2021). Agência Nacional de Cuidados Paliativos. ANCP e Cuidados Paliativos no Brasil. Recuperado de https://www.paliativo.org.br/cuidadospaliativos/cuidados-paliativos-no-brasil

Araújo, M. M. T. \& de Silva, M. J. P. da. (2011). O Conhecimento De Estratégias De Comunicação No the Knowledge About Communication Strategies When Taking Care of the Emotional Dimension in Palliative Care. Texto Contexto Enferm, 3(1), 121-129.

Bakar, M., Capano, E., Patterson, M., McIntyre, B., \& Walsh, C. J. (2020). The Role of Palliative Care in Caring for the Families of Patients With COVID-19. American Journal of Hospice and Palliative Medicine, 37(10), 866-868. https://doi.org/10.1177/1049909120931506

Fausto, J., Hirano, L., Lam, D., Mehta, A., Mills, B., Owens, D., Perry, E., \& Curtis, J. R. (2020). Creating a Palliative Care Inpatient Response Plan for COVID-19-The UW Medicine Experience. Journal of Pain and Symptom Management, 60(1), e21-e26. https://doi.org/10.1016/j.jpainsymman.2020.03.025 
Florêncio, R. S., Cestari, V. R. F., Souza, L. C. de, Flor, A. C., Nogueira, V. P., Moreira, T. M. M., Salvetti, M. de G., \& Pessoa, V. L. M. de P. (2020). Cuidados paliativos no contexto da pandemia de COVID-19: desafios e contribuições. Acta Paulista de Enfermagem, 33, 1-9. https://doi.org/10.37689/actaape/2020ao01886

Fadul, N., Elsayem, A. F., \& Bruera, E. (2021). Integration of palliative care into COVID-19 pandemic planning. BMJ Supportive and Palliative Care, 11(1), 40-44. https://doi.org/10.1136/bmjspcare-2020-002364

Gomes, I. S., \& Caminha, I. D. O. (2014). Guide to systematic review of studies: An option for the methodology of human movement sciences. Movimento, 20(1), 395-411. https://doi.org/10.22456/1982-8918.41542

Heymann, D. L., \& Shindo, N. (2020). COVID-19: what is next for public health? The Lancet, 395(10224), 542-545. https://doi.org/10.1016/S01406736(20)30374-3

Hetherington, L., Johnston, B., Kotronoulas, G., Finlay, F., Keeley, P., \& McKeown, A. (2020). COVID-19 and Hospital Palliative Care - A service evaluation exploring the symptoms and outcomes of 186 patients and the impact of the pandemic on specialist Hospital Palliative Care. Palliative Medicine, 34(9), 1256-1262. https://doi.org/10.1177/0269216320949786

Lovell, N., Maddocks, M., Etkind, S. N., Taylor, K., Carey, I., Vora, V., Marsh, L., Higginson, I. J., Prentice, W., Edmonds, P., \& Sleeman, K. E. (2020). Characteristics, Symptom Management, and Outcomes of 101 Patients With COVID-19 Referred for Hospital Palliative Care. Journal of Pain and Symptom Management, 60(1), e77-e81. https://doi.org/10.1016/j.jpainsymman.2020.04.015

Li, R., Pei, S., Chen, B., Song, Y., Zhang, T., Yang, W., \& Shaman, J. (2020). Substantial undocumented infection facilitates the rapid dissemination of novel coronavirus (SARS-CoV-2). Science, 368(6490), 489-493. https://doi.org/10.1126/science.abb3221

Norris, S. E., Strumph, K., \& Rahmani, N. E. (2020). Pediatric palliative care when COVID-19 positive adults are dying in a children's hospital. Pediatrics, 146(3). https://doi.org/10.1542/PEDS.2020-1570

OPA/OMS (2021). Folha informativa COVID-19 - Escritório da OPAS e da OMS no Brasil. Recuperado de https://www.paho.org/pt/covid19

OMS (2020). Organização Mundial da Saúde. Cuidados Paliativos. https://www.who.int/es/news-room/fact-sheets/detail/palliative-care

Pereira, A., Shitsuka, D., Parreira, F., \& Shitsuka, R. (2018). Método Qualitativo, Quantitativo ou Quali-Quanti. In Metodologia da Pesquisa Científica. https://repositorio.ufsm.br/bitstream/handle/1/15824/Lic_Computacao_Metodologia-Pesquisa-Cientifica.pdf?sequence=1.

Powell, V. D., Silveira, M. J. What Should Palliative Care's Response Be to the COVID-19 Pandemic? J Pain Symptom Manage. 2020 60(1):e1-e3. 10.1016/j.jpainsymman.2020.03.013.

Palliative, U., Consult, C., \& Only, T. (2020). COVID-19 Articles Fast Tracked Articles What Should Palliative Care's Response Be to the COVID-19 Pandemic? 60(1), 1-3.

Ribeiro, A. P., Oliveira, G. L., Silva, L. S., \& Souza, E. R. de. (2020). Saúde e segurança de profissionais de saúde no atendimento a pacientes no contexto da pandemia de Covid-19: revisão de literatura. Revista Brasileira de Saúde Ocupacional, 45, 1-12. https://doi.org/10.1590/2317-6369000013920

Silva, M. P. B., et al (2021). As contribuições da equipe multiprofissional na atenção básica à saúde frente aos cuidados paliativos. Research, Society and Development, 10 (4), e40210413887. https://doi.org/10.33448/rsd-v10i4.13887

Santos, C. G. da S., Tavares, A. P. dos S., Martins, C. T., Barros, J. N., Silva, A. M. M., Lotaif, L., \& Souza, J. V. L. (2020). Cuidado paliativo renal e a pandemia de Covid-19. Brazilian Journal of Nephrology, 42((2 suppl 1)), 44-46. https://www.scielo.br/pdf/jbn/v42n2s1/pt_2175-8239-jbn-42-02-s010044.pdf

SANTOS CMC, et al. A estratégia PICO para a construção da pergunta de pesquisa e busca de evidências. Revista Latino-Americana de Enfermagem, 2007; 15: 508-511. 10.1590/S0104-11692007000300023.

Ting, R., Edmonds, P., Higginson, I. J., \& Sleeman, K. E. (2020). Palliative care for patients with severe covid-19. The BMJ, 370, 1-4. https://doi.org/10.1136/bmj.m2710

Tritany, É. F., Filho, B. A. B. de S., \& de Mendonça, P. E. X. (2021). Strengthening palliative care during the covid-19 pandemics. Interface: Communication, Health, Education, 25, 1-14. https://doi.org/10.1590/Interface.200397

WHO. World Health Organization. Director - Gernal's opening remarks at the media briefing on COVID-19 (2020). Geneva: World Health Organization. Recuperado de: https://www.who.int/dg/speeches/detail/who-director-general-s-opening-remarks-at-the-media-briefing-on-covid-19---11-march-2020

World Health Organization (2021). Manutenção de serviços de saúde essenciais durante o surto COVID-19. https://www.who.int/emergencies/diseases/novelcoronavirus-2019/related-health-issues?gclid=Cj0KCQiAgomBBhDXARIsAFNyUqP14BqIzj5ei2hjdtD8Ie_Llqp1JQcHY-jVyjuvTFh6W4qNJS3nanUaAu-

0EALw_wcB

Yeung, S., Wong, S., Zhang, D., Wing, R., Sit, S., Hon, B., Yip, K., Chung, R. Y., Ka, C., Wong, M., Cheong, D., Chan, C., Sun, W., \& Kwok, K. O. (2020). Impact of COVID-19 on loneliness, mental health, and health service utilisation: a prospective cohort study of older adults with multimorbidity in primary care. British Journal of General Practice, November, 817-824.

Zhang, J., Feng, B., Wu, Y., Xu, P., Ke, R., \& Dong, N. (2021). The effect of human mobility and control measures on traffic safety during COVID-19 pandemic. PLoS ONE, 16, 493-497. https://doi.org/10.1371/journal.pone.0243263 